\title{
Numerical computation of turbulent gas-particle flow in a 90 degree bend: comparison of two particle modelling approaches
}
K. Mohanarangam ${ }^{1}$
Z. F. $\operatorname{Tian}^{2}$
J. Y. $\mathrm{Tu}^{3}$

(Received 3 August 2006; revised 20 December 2007)

\begin{abstract}
A numerical study into the physical characteristics of dilute gasparticle flows over a square-sectioned $90^{\circ}$ bend is reported. Two approaches, namely the Lagrangian particle tracking model and Eulerian two fluid model are employed to predict the gas-particle flows. Renormalization Group based $k-\epsilon$ model is used as the turbulent closure for both the approaches; however, for the Eulerian model, additional transport equations are solved to account for the combined gas-particle interactions and the turbulence kinetic energy of the particulate phase.
\end{abstract}

See http://anziamj.austms.org.au/ojs/index.php/ANZIAMJ/article/view/69 for this article, (c) Austral. Mathematical Soc. 2007. Published December 27, 2007. ISSN 1446-8735 
In comparison to both Eulerian-Eulerian and Eulerian-Lagrangian approaches the former was found to yield closer agreement with the measured values. The Eulerian-Lagrangian approach obtains finer details of the behaviour of particles, while Eulerian-Eulerian model can be used for industrial scale problems. All the simulated results are compared against the experimental finding of Kliafas and Holt (1987).

\section{Contents}

1 Introduction

C743

2 Computational methods

C744

2.1 Mathematical model based on a two fluid approach . . . . C744

2.2 Mathematical model based on Lagrangian particle tracking model

3 Numerical procedure

3.1 Eulerian approach . . . . . . . . . . . . . . . .

3.2 Lagrangian approach . . . . . . . . . . . . . . . C C748

4 Results and discussion

4.1 Mean velocities . . . . . . . . . . . . . . . C750

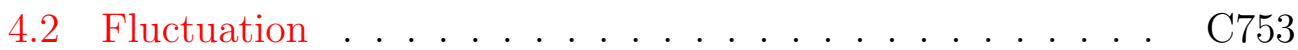

5 Conclusion

C754

References

C756 


\section{Introduction}

A lot of engineering applications employ dilute two-phase flows, ranging from pulverised coal fired combustion systems to drug delivery systems used in biomedical fields. An efficient design of these systems will usually require an in depth understanding on how the two phases behave under varying conditions. Numerical simulations, a key alternative to decipher these complex processes makes use of the current computational power and efficiency to provide an attractive answer and a powerful exploration tool for both institutional and industry researchers.

Two major techniques used for the simulation of two phase flows are the Eulerian-Eulerian two fluid model and the Lagrangian particle tracking model. In the Eulerian approach the gas and particle phases are considered as interpenetrating continua coupled together by exchange co-efficient(s). In the Lagrangian approach, each particle is tracked within the computational domain, which eases the implementation of the exchange co-efficient(s).

This article simulates the dilute gas-particle flow in a square sectioned $90^{\circ}$ bend using both a Lagrangian particle tracking model (DRW model) and an Eulerian two fluid model [9]. The current study is in line with that of Durst et al. [2] and Adeniji-Fashola and Chen [1] who also compared both numerical approaches. Durst et al. [2] introduced a simple drag force interaction between the two phases for both the approaches and summarized that it was more of a test of the numerics rather than the actual physics. Whereas the work of Adeniji-Fashola and Chen [1] was a bit more advanced than its counterpart, they still had problems predicting concentration and also the specification of the boundary condition for the particle trajectory. Both the above works were performed on a simpler geometry of a vertical channel flow.

The current work more or less tries to overcome the problems mentioned above, but with a far more complex geometry; that is, turbulent flow in a 
$90^{\circ}$ bend. Flows in curved ducts are usually quite complex and are characterized by a stress field with stabilizing effects near to the inner radius and destabilizing effects close to the outer radius [5]. Numerical simulations help us access fundamental understanding of the two phases in the presence of particles. The simulation results of both the numerical approaches are compared against the experimental data [6], and a detailed discussion about their relative performance and also the shortcomings of the Lagrangain model to predict the particle fluctuation using a commercial code along with its computational expense is exemplified.

\section{Computational methods}

\subsection{Mathematical model based on a two fluid approach}

The modified Eulerian two fluid model developed by Tu [11, 9] used in this study considers the gas and particle phases as two interpenetrating continua. Here, a two way coupling is achieved between the continuum gas and particle phase.

In order to better represent the combined gas-particle behavior, the transport equation for the gas-particle covariance $\overline{u_{g}^{\prime i} u_{g}^{\prime i}}$ governing the gas-particle correlation is

$$
\frac{\partial}{\partial x_{j}}\left[\rho_{p}\left(u_{g}^{j}+u_{p}^{j}\right) k_{g p}\right]=\frac{\partial}{\partial x_{j}} \rho_{p}\left(\frac{\nu_{g t}}{\sigma_{g}}+\frac{\nu_{p t}}{\sigma_{p}}\right) \frac{\partial k_{g p}}{\partial x_{j}}+P_{j g p}-\rho_{p} \varepsilon_{g p}-\Pi g p .
$$

This is obtained by subtracting the Favre-averaged momentum equation from the time dependent momentum equation for both the gas and particle phases and the resulting equation for the gas fluctuating velocity being multiplied by the fluctuating flux $\rho_{p} u_{p}^{i}$, and the equation for the particulate fluctuating 
velocity multiplied by $u_{g}^{\prime i}$. Summation of the two equations and subsequent time-averaging yields the $\overline{u_{g}^{\prime i} u_{g}^{\prime i}}$ equation.

The turbulence production by the mean velocity gradients of two phases is

$$
\begin{aligned}
P_{j g p}=\left\{\rho_{p}\left(v_{g t} \frac{\partial u_{g}^{i}}{\partial x_{j}}+v_{p t} \frac{\partial u_{p}^{i}}{\partial x_{i}}\right)-\frac{2}{3} \rho_{p} \delta_{i j} k_{g p}\right. \\
\left.\quad-\frac{1}{3} \bar{\rho}_{p} \delta_{i j}\left(v_{g t} \frac{\partial u_{p}^{k}}{\partial x_{k}}+v_{p t} \frac{\partial u_{p}^{k}}{\partial x_{k}}\right)\right\}\left(\frac{\partial u_{g}^{i}}{\partial x_{j}}+\frac{\partial u_{p}^{i}}{\partial x_{j}}\right) .
\end{aligned}
$$

The interaction term between the two phases takes the form

$$
\Pi_{g p}=\frac{f}{2 t_{p}} \rho_{p}\left[(1+m) 2 k_{g p}-2 k_{g}-m 2 k_{p}\right]
$$

here $m$ is the mass ratio of the particle to the gas, $m=\frac{\rho_{p}}{\rho_{g}}$. The dissipation term due to the gas viscous effect is modeled by

$$
\varepsilon_{g p}=\varepsilon_{g} \exp \left(-B_{e} t_{p} \frac{\varepsilon_{g}}{k_{g}}\right),
$$

where $k_{g}, \varepsilon_{g}, \varepsilon_{g p}, t_{p}$ are the gas turbulent kinetic energy, gas eddy dissipation, combined gas-particle eddy dissipation and particle relaxation time respectively. $B_{e}$ is a constant and takes a value of 0.4 [10].

\subsection{Mathematical model based on Lagrangian particle tracking model}

A generic CFD commercial code, FLUENT, solves the equations of mass and momentum under steady state conditions to yield the gas phase velocity profiles. The mathematical model describing the gas phase is the same as for the two fluid approach. Tian et al. [8] gives more information about the model. 


\section{Numerical procedure}

The simulated results from both the Lagrangian particle tracking and Eulerian two fluid models are compared against the benchmark experimental data of [6] for a gas-particle flow in a $90^{\circ}$ bend. The particles are made of glass with a material density $\rho_{p}=2990 \mathrm{~kg} / \mathrm{m}^{3}$ and diameter size of $50 \mu \mathrm{m}$. A nonorthogonal boundary fitted coordinate grid is employed for the study. The sample grid used for the computations is illustrated in Figure 1. The computational domain starts $10 D$ upstream from the bend entrance and extends up to $12 D$ downstream from the bend exit; there is also a uniform distribution of 45 control volumes placed at every two degree interval along the bend.

The governing transport equations are discretized using finite volumes. The equations are solved on a nonstaggered grid system. Third order QUICK scheme is used to approximate the convective terms, while second order accurate central difference scheme is adopted for the diffusion terms. The velocity correction is realized to satisfy continuity through SIMPLE algorithm, which couples velocity and pressure. Uniform velocity is imposed at the top inlet plane of the bend with wall boundary conditions imposed on the top and bottom and also along the sides of the $90^{\circ}$ square duct. The computational domain remains the same for both the approaches.

\subsection{Eulerian approach}

In the Eulerian model, all the governing equations for both gas and particle phase are solved sequentially at each iteration. The solution process starts by solving the momentum equations of the gas phase followed by the pressure correction through continuity. This is then followed by solution of turbulence equations for the gas phase, whereas the solution process for the particle phase starts by solving the momentum equations followed by the 


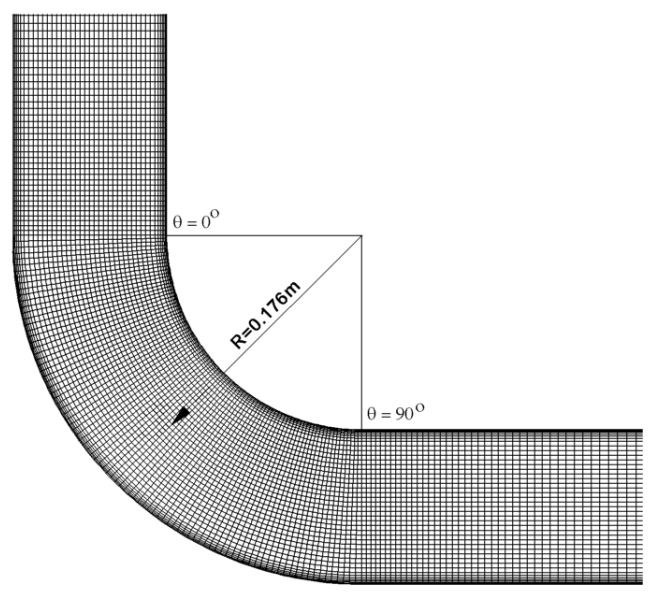

(a) Bend

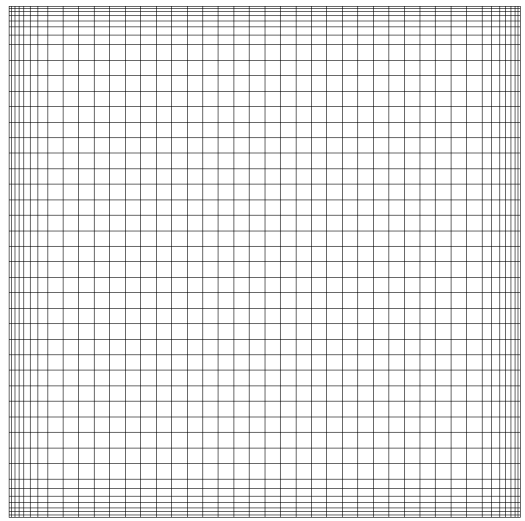

(b) Bend Span

Figure 1: Computational Domain and grids of $90^{\circ}$ bend. 
concentration and then the gas-particle turbulence interaction and ends by solving the turbulence equation for the particulate phase. At each global iteration, each equation is iterated, typically three to five times, using a strongly implicit procedure (SIP). The above solution process is marched towards a steady state and is repeated until a converged solution is obtained.

In order to match the experimental conditions, uniform velocity, $U_{b}=$ $52.19 \mathrm{~m} / \mathrm{s}$, for both gas and particle phases are imposed at the top inlet $1 \mathrm{~m}$ away from the bend entrance, which corresponds to a Reynolds number of $3.47 \times 10^{5}$. The inlet turbulence intensity is $1 \%$ whereas the particles are taken to be glass spheres of density $\rho_{s}=2,990 \mathrm{~kg} / \mathrm{m}^{3}$ and size $50 \mu \mathrm{m}$; with the inlet particulate bulk density $\rho_{p \text {,in }}=1.8 \times 10^{-4}$. The corresponding particulate loading and volumetric ratios are $1.5 \times 10^{-4}$ and $6 \times 10^{-8}$ respectively, for which the particle suspension is very dilute.

At the outflow the normal gradient for all dependent variables are set to zero. A no-slip boundary condition is employed along the wall for the gas phase and for the particulate phase Eulerian boundary conditions are specified to represent the particle-wall momentum transfer [11].

\subsection{Lagrangian approach}

The particle transport using the DRW model was computed from the converged solution of the gas flow. For the DRW model, a total of 50000 particles were released from ten uniformly distributed points across the inlet and they were individually tracked within the bend. The independence of statistical particle phase prediction from the increase of particle number was tested using 100,000 particles. The difference of the maximum positive velocities of for both the cases was less than $1 \%$. The boundary condition for the Lagrangian model is in accordance to the Eulerian model except for particles at the wall where a 'reflective' type boundary condition is used.

All the calculations were performed on a PC workstation with a $3.0 \mathrm{GHz}$ 
CPU and 1 Gbyte of RAM. To investigate the computational performance for Lagrangian and Eulerian approaches, CPU times for both of the methods were recorded. The CPU times described here comprise both the convergence time for the gas phase and the calculation time for the particles phase. The computational time required for the Lagrangian approach is almost 20 times the time required for its counterpart Eulerian approach.

\section{Results and discussion}

The mean quantities of both gas and solid phases are compared with two different numerical approaches. In order to carry out this prognostic approach, the models were validated against the well established experimental result [6]. An important, dimensionless scaling parameter in fluid-particle flow is the Stokes number (St), which is defined as the ratio between the particle relaxation time to a time characteristic of the fluid motion, that is, $\mathrm{St}=t_{p} / t_{s}$. This determines the kinetic equilibrium of the particles with the surrounding gas. The system relaxation time, $t_{s}$, in the Stokes number definition is derived from the characteristic length $D$ and the characteristic velocity of the system under investigation, which in our case is the inlet bulk velocity $U_{b}$; that is, $t_{s}=D / U_{b}$. A small stokes number $(\mathrm{St} \ll 1)$ signifies that the particles are in near velocity equilibrium with the carrier fluid. For larger stokes number $(\mathrm{St} \gg 1)$ particles are no longer in equilibrium with the surrounding fluid phase, which will be explained in the later sections. In this section, the mean quantities of both the gas and particulate phases, that is, their velocity and fluctuation distributions along the bend are compared, as mean quantities are of utmost interest in engineering applications. The results obtained from both approaches are recorded from the mid plane of the duct geometry. All the values reported here (unless or otherwise stated) are normalized using the inlet bulk velocity $U_{b}$. 


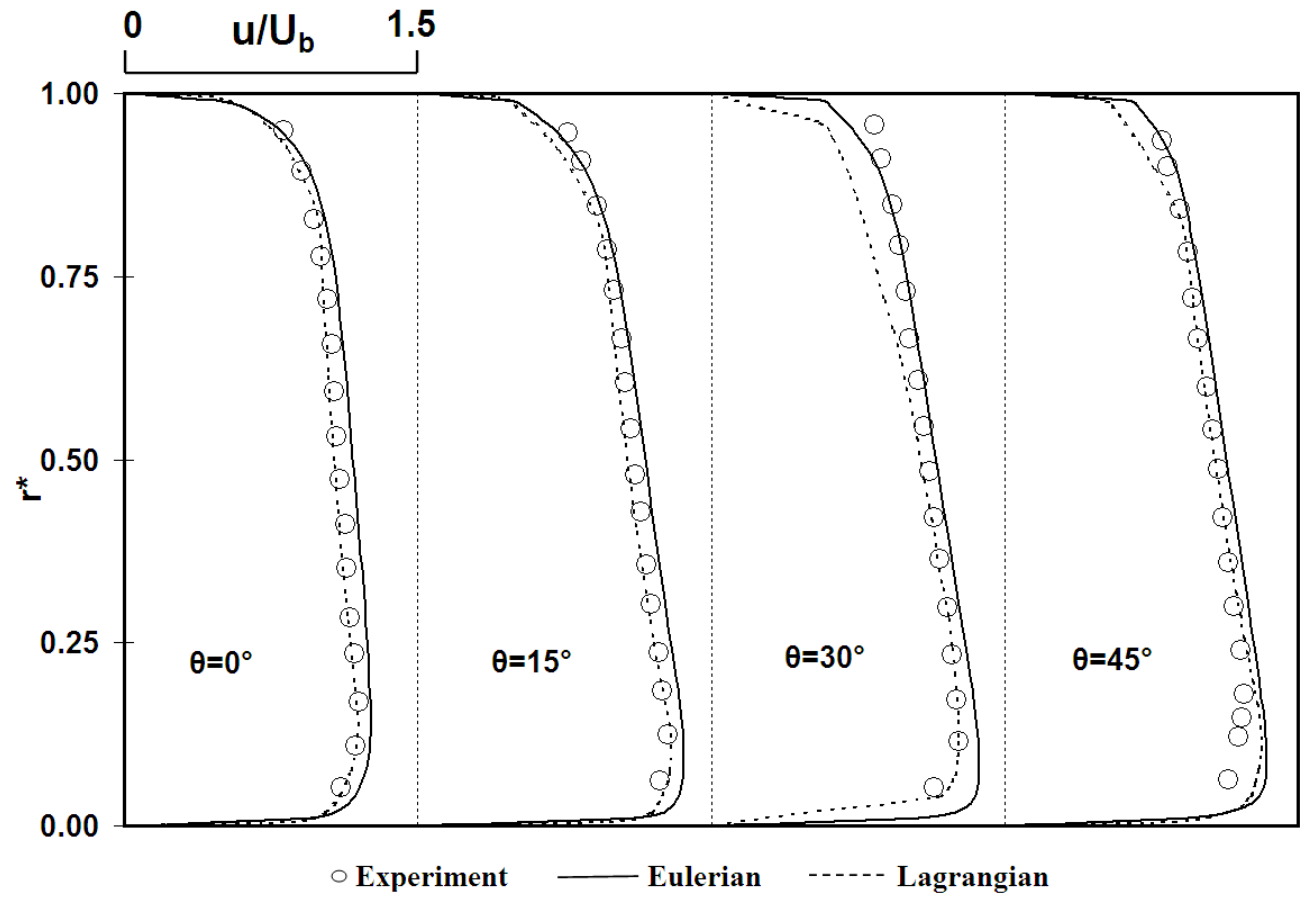

Figure 2: Mean streamwise gas velocities along the bend.

\subsection{Mean velocities}

Figure 2 shows the comparison of the numerical results against the experimental data for mean streamwise gas velocity along various sections of the bend. A good quantitative comparison is achieved with both numerical models against the data from Kliafas and Holt [6].

Figure 3 shows the comparison of mean particle velocities; there is generally a good agreement between the experimental and predicted data using both Eulerian and Lagrangian numerical approaches. The Stokes number for the experimental case was found to be about 12.87 (that is, St $\gg 1$ ) with 


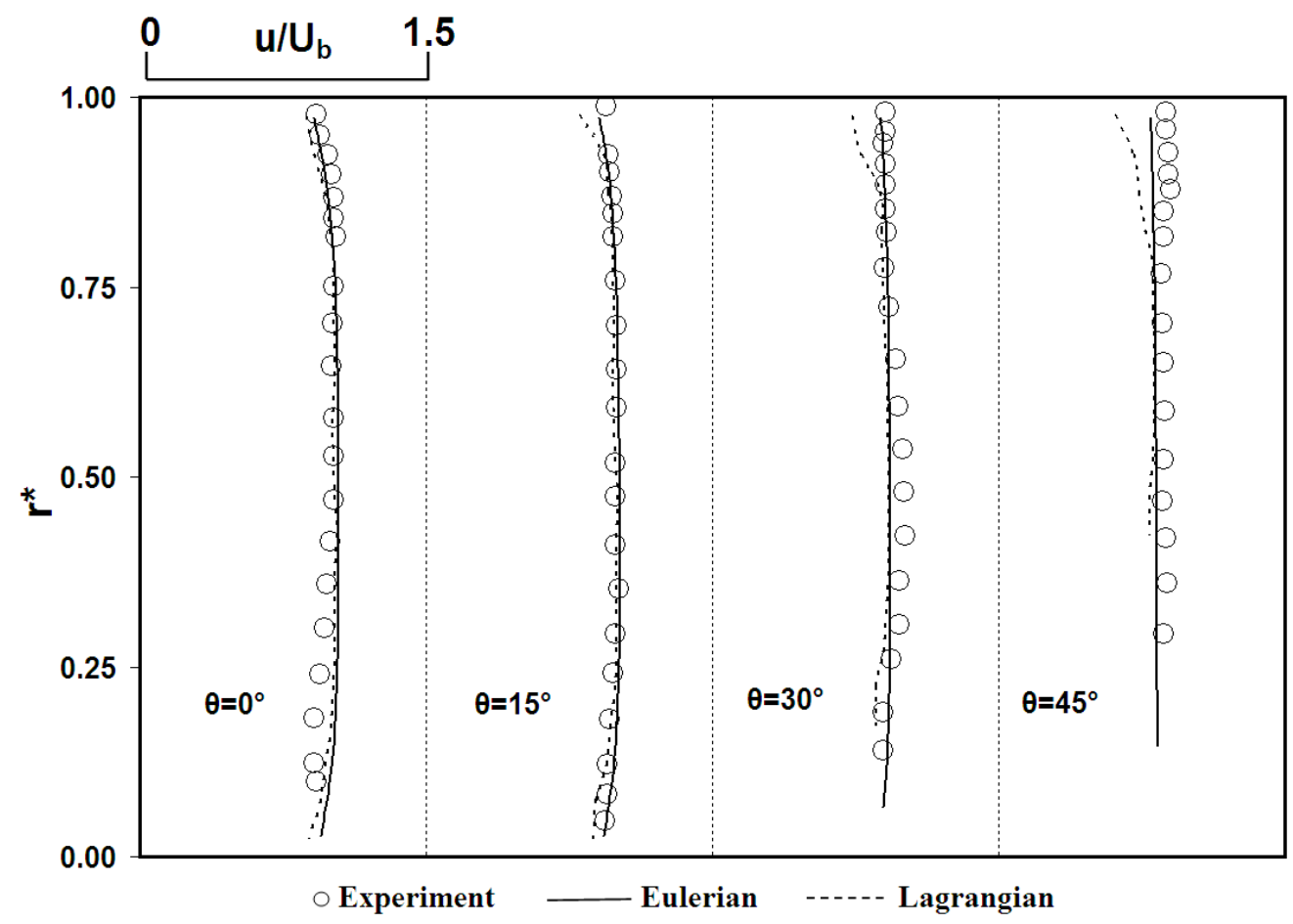

FiguRE 3: Mean streamwise particle velocities along the bend.

flatter profiles noted for the dispersed phase. This proves that they are not affected by gas pressure gradients. The particle velocities are generally lower than the fluid velocities, which is similar to the observations made by Kulick et al. [7] where the particles in the channel flow show a negative slip velocity due to cross-stream transport. In order to better understand the particle behavior around the carrier gas phase, further simulations for various Stokes numbers are presented using Eulerian two fluid formulation against the carrier gas phase velocity. Figure 4 shows that particles act as 'gas tracers' for a Stokes number of 0.01 as they are found to be fully in equilibrium with the carrier phase and this phenomenon becomes less pronounced as the Stokes 


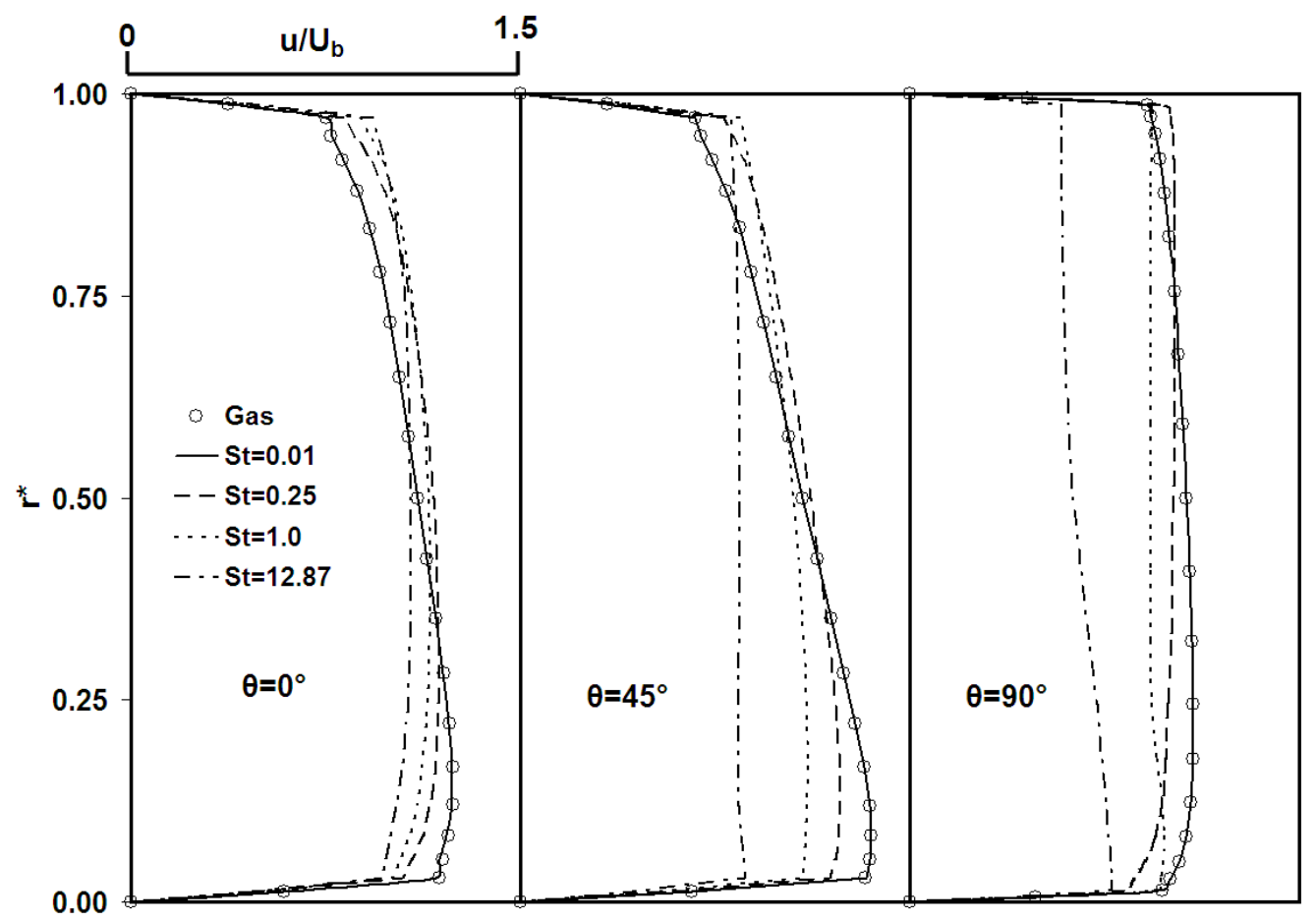

Figure 4: Streamwise velocities along the bend for varying Stokes number.

number is increased. From the observations of 0 and 45 degree bend sections, there exists a positive slip velocity between the particulate and the gas phase at the outer walls, along with the velocities of the gas peaking at the inner walls due to the presence of a favorable pressure gradient. This 'gas tracing' phenomena of the particles become less pronounced as we approach the bend exit, as the flow regains the energy it lost due to slip. For flows with St $\geq 1$ the positive slip velocity between the particle and gas velocity keeps decreasing along with the bend radius and turns negative at the bend exit where the gas leads the particle. This is attributed to the particles not able to keep up with the gas due to its own inertia in addition to its energy loss attributed towards particle wall collisions. 


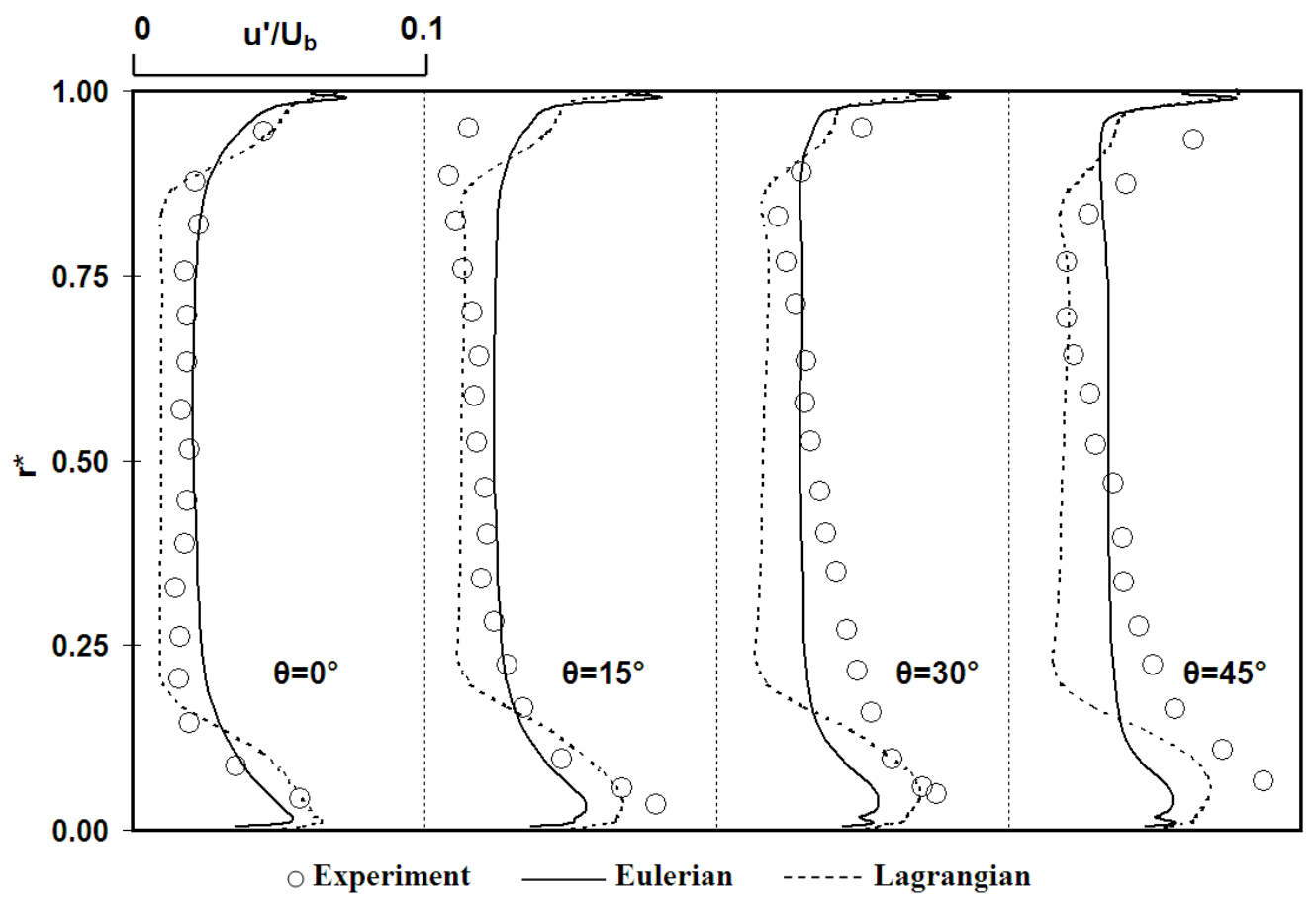

FigURE 5: Measurements and calculations of mean streamwise intensity profiles of gas.

\subsection{Fluctuation}

The predictions of streamwise turbulence intensity of the carrier phase in the Eulerian and Lagrangian models are compared in Figure 5, wherein a very small Stokes number of particle phase is used as the seed. High turbulence intensity near the walls is due to high shear rate, when compared to the core region of the flow. There is a general under prediction by both methods towards the outer wall in the 30 and 45 degree sections. Figure 6 illustrates the computed particle fluctuation compared with the available experimental results. There is a remarkable qualitative agreement with the experimental 
results against the Eulerian two fluid model except for some parts within the 30 degree section. The particle fluctuating velocities are found to be considerably larger than the gas phase, and this may be attributed to the cross stream mixing [3], this phenomena reported in previous studies, is a direct consequence of transport of inertial particles across regions of mean shear [4]. Fessler and Eaton [4] infer that fluctuating velocity of the particles in the wall-normal direction are consistently lower than the fluid, as flow directions with low mean shear are encountered there.

However, there is a serious under-prediction of particle fluctuation using the Lagrangian model of particle tracking and hence not shown for comparison with the reported literature or the Eulerian model. The poor agreement may be attributed to the weak coupling between carrier and dispersed phases in comparison to Eulerian two fluid model where there exists a strong coupling between the two phases, incorporated by solving additional transport equations for turbulent kinetic energy and eddy dissipation for combined gas and particle phases as detailed in the previous section. Another possible reason for this discrepancy is the inability of the Lagrangian particle tracking model to simulate interactions with large scale, instantaneous flow structures [3].

\section{Conclusion}

The current study investigated the physical characteristics of the dilute particle laden flow in a square sectioned $90^{\circ}$ bend through the comparison made by two approaches: Eulerian two fluid model and Lagrangian particle tracking model (DRW model). A significant amount of work was undertaken in this article to provide an in-depth understanding of the particle response to turbulence under the influence of its own inertia (Stokes number). The computational results obtained by both the Lagrangian model and the Eulerian model were compared against the benchmark experimental data. The 


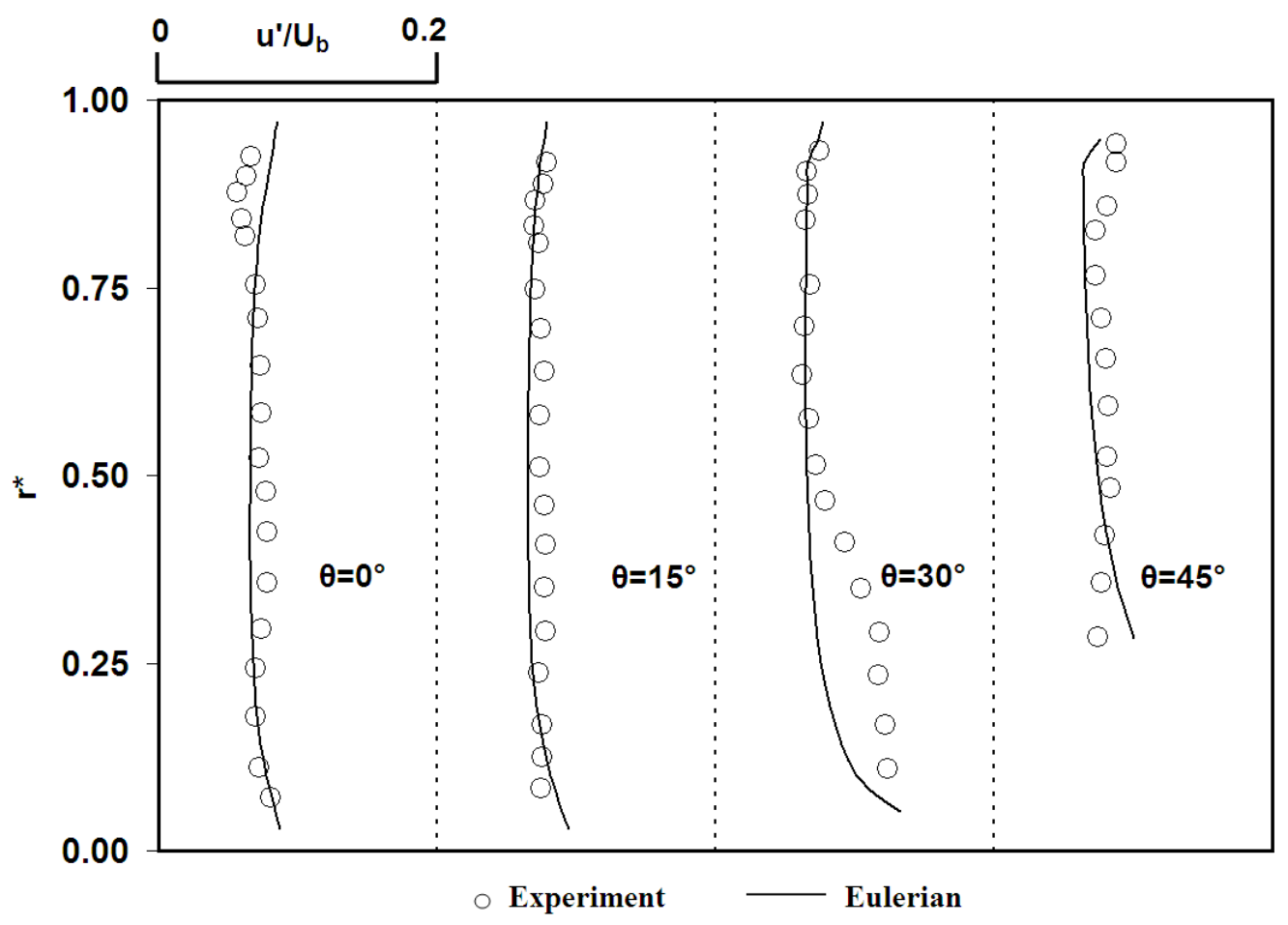

FiguRE 6: Measurements and calculations of mean streamwise intensity profiles of particle. 
results reveal that both approaches provided reasonably good comparison for gas and particle velocities together with the fluctuation for the gas phase. Despite the fact that the particle fluctuation using the Eulerian model showed good comparison with the experimental data, there was a severe under prediction of this quantity using the Lagrangian particle tracking model. More computational mesh and time is required for Lagrangian particle tracking model in comparison to Eulerian model. For complex flows such as in this study, the Eulerian approach is still far more attractive than its Lagrangian counterpart.

Acknowledgements The first author was supported by IPRS research scholarship and the financial support provided by the Australian Research Council (project LP0347399) is gratefully acknowledged.

\section{References}

[1] A. Adeniji-Fashola and C. P. Chen. Modelling of confined turbulent fluid-particle flows using eulerian and lagrangian schemes. Int. J. Heat Mass Transfer, 3(4):691-701, 1990. doi:10.1016/S0307-904X(98)00007-9. C743

[2] F. Durst, D. Milojevic, and B. Schonung. Eulerian and lagrangian predictions of particulate two-phase flows :a numerical study. Appl. Math. Model, 8(2):101-115, 1984. doi:10.1016/0307-904X(84)90062-3. $\mathrm{C} 743$

[3] J. R. Fessler and J. K. Eaton. Particle response in a planar sudden expansion flow. Expl Thermal and Fluid Sci, 15:413-423, 1997. doi:10.1016/S0894-1777(97)00010-1. C754

[4] J. R. Fessler and J. K. Eaton. Turbulence modification by particles in a backward-facing step flow. J. Fluid Mech, 394:97-117, 1999. http: 
//journals . cambridge.org/action/displayAbstract?aid=15369. C754

[5] J. A. C. Humphrey, J. H. Whitelaw, and G. Yee. Turbulent flow in a square duct with strong curvature. J. Fluid Mech., 103:443-463, 1981. doi:10.1017/S0022112081001419. C744

[6] Y. Kliafas and M. Holt. Ldv measurements of a turbulent air-solid two-phase flow in a 90 degree bend. Exp. Fluids, 5:73-85, 1987. doi:10.1007/BF00776177. C744, C746, C749, C750

[7] J. D. Kulick, J. R. Fessler, and J. K. Eaton. Particle response and turbulence modification in fully developed channel flow. J. Fluid Mech., 277:109-134, 1994. doi:10.1017/S0022112094002703. C751

[8] Z. F. Tian, J. Y. Tu, and G. H Yeoh. Numerical simulation and validation of dilute gas- particle flow over a backward-facing step. Aerosol Sci. Technol., 39:319-332, 2005. doi:10.1080/027868290930961. $\mathrm{C} 745$

[9] J. Y Tu. Computational of turbulent two-phase flow on overlapped grids. Numer. Heat Transfer Part B Fundam, 32:175?195, 1997. doi:10.1080/10407799708915004. C743, C744

[10] J. Y. Tu and C. A. J. Fletcher. An improved model for particulate turbulence modulation in confined two-phase flows. Int. Commun. Heat Mass Transfer, 21(6):775-783, 1994. doi:10.1016/0735-1933(94)90031-0. C745

[11] J. Y. Tu and C. A. J. Fletcher. Numerical computation of turbulent gas-solid particle flow in a 90 degree bend. AIChE Journal, 41(10):2187-2197, 1995. doi:10.1002/aic.690411003. C744, C748 


\section{Author addresses}

1. K. Mohanarangam, School of Aerospace, Mechanical and Manufacturing Engineering, Royal Melbourne Institute of Technology, Bundoora, Victoria 3083 , Australia. mailto: jiyuan.tu@rmit.edu.au

2. Z. F. Tian, School of Aerospace, Mechanical and Manufacturing Engineering, Royal Melbourne Institute of Technology, Bundoora, Victoria 3083 , Australia.

3. J. Y. Tu, School of Aerospace, Mechanical and Manufacturing Engineering, Royal Melbourne Institute of Technology, Bundoora, Victoria 3083 , Australia. 